\title{
Interferon-beta verstärkt die Vitamin-D-Synthese bei Sonnenlicht
}

Fragestellung: Ist eine Interferon-beta-Behandlung mit unterschiedlichen Vitamin-D-Spiegeln im Serum assoziiert und wirkt sich dies auf die Schubrate aus?

Hintergrund: Nach wie vor ist die Rolle von Vitamin D bei Autoimmunerkrankungen, aber auch bei degenerativen Erkrankungen wie dem Morbus Parkinson und bei vaskulären Erkrankungen wie Schlaganfällen unklar. Einzelne Studien ergaben in der Vergangenheit Hinweise darauf, dass niedrige Vitamin-DSpiegel mit einer erhöhten Krankheitsaktivität bei Autoimmunerkrankungen assoziiert sind. Dies war insbesondere bei den kindlichen und juvenilen Formen der MS gezeigt worden. Insgesamt ist die Datenlage jedoch uneinheitlich, sodass bisher nicht grundsätzlich davon ausgegangen werden kann, dass die Substitution von Vitamin D mit einem therapeutischen Effekt bei Autoimmunerkrankungen verbunden ist.

In einer australischen Studie wurde nun überprüft, inwieweit die Gabe von Interferon-beta mit höheren Vitamin-D-Spiegeln vergesellschaftet ist und inwieweit mögliche Unterschiede wiederum mit einer Änderung der Schubrate assoziiert sind.

Patienten und Methodik: In einer prospektiven Kohortenstudie mit 178 Patienten, die an einer MS erkrankt waren, wurde im Zeitraum von 2002 bis 2005 zweimal pro Jahr der VitaminD-Spiegel (25-Hydroxyvitamin D, 25-OH-D) gemessen. Parallel dazu wurden relevante klinische Faktoren sowie Behandlungen erhoben und dokumentiert. Die Studie erfolgte im Süden von Tasmanien (südlich von Australien), wo die Sonneneinstrahlung im Sommer und Winter stark variiert. Der Serumspiegel-Vergleich des Vitamin-D erfolgte auch zwischen Sommer und Winter und erlaubte damit auch eine Assoziation mit der Dauer der Sonnenexposition.

Ergebnisse: Patienten unter einer Interferon-Therapie zeigten einen signifikant höheren Vitamin-D-Spiegel als Patienten ohne Interferon-Therapie. Der Serumspiegel war ferner abhängig von der Sonneneinstrahlung, sodass Patienten im Winter einen deutlich niedrigeren Vitamin-D-Spiegel aufwiesen als im Sommer. Auch hier zeigte sich, dass im Sommer der Vitamin-D-Spiegel unter der Interferon-Therapie deutlich höher war. Insgesamt schwankte der Vitamin-D-Spiegel zwischen den Jahreszeiten im mittleren Bereich zwischen 35 und $49 \mathrm{nmol} / \mathrm{l}$ (im Winter) bis zu $70 \mathrm{nmol} / \mathrm{l}$ (im Sommer). Die Schubrate war signifikant unterschiedlich zwischen den Patienten, die ein Interferon-Präparat

Stewart N, Simpson S Jr, van der Mei I et al. Interferon- $ß$ and serum 25-hydroxyvitamin D interact to modulate relapse risk in MS. Neurology 2012; 79: $254-60$ erhielten und den Patienten, die nicht unter Therapie waren. Deutlich größer war jedoch der Unterschied, wenn der Vitamin-D-Spiegel über $50 \mathrm{nmol} / \mathrm{l}$ lag. Hier lag die Schubrate bei Patienten ohne
Therapie bei 1,31; unter Interferon-Therapie jedoch bei 0,48 . Bei Patienten unter Interferon-Therapie zeigten sich ebenfalls Unterschiede in Abhängigkeit des Vitamin-D-Spiegels. Bei einem Vitamin-D-Spiegel unter $50 \mathrm{nmol} / \mathrm{l}$ lag die Schubrate bei 2,01; während sie bei einem Spiegel über $50 \mathrm{nmol} / 1$ nur bei $0,58 \mathrm{lag}$. Alle Unterschiede waren statistisch signifikant.

Schlussfolgerungen: Die Studie zeigt zum einen höhere VitaminD-Spiegel unter einer Interferon-Therapie, zum anderen aber auch, unabhängig von einer Interferon-Therapie, dass Patienten mit hohen Vitamin-D-Spiegeln weniger Schübe haben, als $\mathrm{Pa}$ tienten mit niedrigeren Vitamin-D-Spiegeln. Den besten Schutz haben offensichtlich Patienten unter einer Interferon-Therapie, die hohe Vitamin-D-Spiegel aufweisen. Die Autoren folgern hier unter anderem, dass über spezifische metabolische Wege die immunmodulativen Effekte von Interferon-beta-Präparaten über eine Erhöhung des Vitamin-D-Spiegels vermittelt werden.

\section{- Kommentar von Volker Limmroth, Köln}

\section{Vitamin D bleibt auf der Agenda}

Die prospektive Studie ist zweifellos interessant und gibt der Thematik zu Vitamin-D als wichtiger Komponente bei der Vermittlung autoimmunologischer Erkrankungen neue Nahrung. Zwar ist die Studie prospektiv durchgeführt worden, dennoch bleibt die Zahl der beobachteten Patienten relativ klein. Von den 178 untersuchten Patienten hatten $63 \%$ eine InterferonMedikation erhalten, 37\% nicht, sodass die Gruppen recht unterschiedlich waren. Auffällig bleiben dennoch die großen Schwankungen zwischen den Jahreszeiten, die zu einem Abfall von bis zu $50 \%$ beziehungsweise zu einem Anstieg über Sonnenexposition von über $100 \%$ führen können. Streng genommen müsste man hier erkennen können, dass die Schubrate insbesondere im Winter deutlich zunehmen müsste, während sie im Sommer dann niedriger wäre. Diese Frage beziehungsweise Auswertung bleibt die Studie allerdings schuldig. Insgesamt bleibt das Thema Vitamin D daher auf der Agenda.

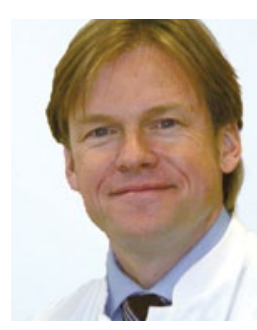

Prof. Dr. med. Volker Limmroth, Köln-Merheim

Chefarzt der Klinik für Neurologie und Palliativmedizin Köln-Merheim E-Mail: LimmrothV@kliniken-koeln.de 